\title{
A BIMODAL MODEL OF THE SOLAR WIND SPEED
}

\author{
W.A.COLES \\ E.C.E.Dept., University of California, La Jolla, CA 92093-0407 \\ Phone 619-534-2703; Fax 619-534-2486; email bcoles@ucsd.edu
}

December 1, 1996

\begin{abstract}
.
Until the ULYSSES spacecraft reached high latitude, the only means for measuring the solar wind velocity in the polar regions was from radio scattering observations (IPS), and these remain the only way to measure the velocity near the sun. However, IPS, like many remote sensing observations, is a "line-of-sight" integrated measurement. This integration is particularly troublesome when the line-of-sight passes through a fast stream but that stream does not occupy the entire scattering region. Observations from the HELIOS spacecraft have shown that the solar wind has a bimodal character which becomes more pronounced near the sun. Recent observations from ULYSSES have confirmed that this structure is clear at high latitudes even at relatively large solar distances. We have developed a method of separating the fast and slow contributions to an IPS observation which takes advantage of this bimodal structure. In this paper I will describe the technique and its application to IPS observations made using the receiving antennas of the EISCAT incoherent backscatter radar observatory in northern Scandinavia.
\end{abstract}

Key words: solar wind - plasma — interplanetary scintillation

\section{Introduction}

Evidence is accumulating that the state of the solar wind is bimodal [Schwenn, 1990; Axford, this volume; Axford and McKenzie, in preparation]. We will refer to these two states as "fast streams" and "slow wind". The fast streams appear to emanate from regions of the corona with unipolar magnetic field (coronal holes) which are dark both in soft X-ray emission and white-light polarized brightness measurements. Coronal holes are cooler and less dense than the corona over closed field regions. Fluctuations in the fast wind are dominated by outward propagating Alfvéen waves of relatively high amplitude [Marsch and Tu, 1990].

The slow wind is observed above regions of the corona with complex magnetic topology such as active regions and helmet streamers. It is not clear whether it comes from the boundaries of coronal holes or whether it "leaks out" from unresolved open field regions within larger closed field regions. The slow wind is less homogeneous; it is denser and threaded with magnetic field reversals. Fluctuations in the slow wind are not as Alfvénic as those in the fast wind, consisting largely of convected pressure balance structures [Marsch and Tu, 1990]. Waves are observed to propagate both outwards and inwards.

Plasma observations from the Helios spacecraft close to the sun, have 


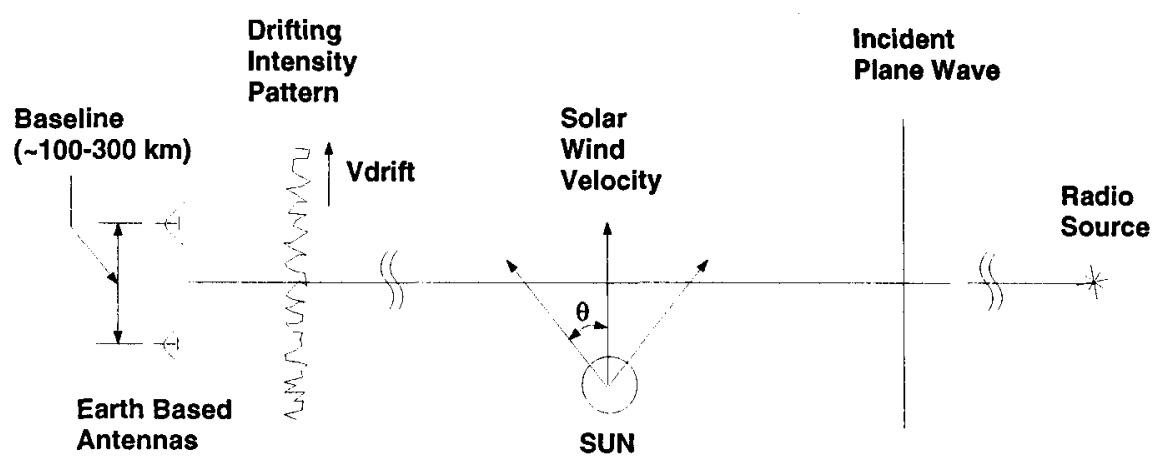

Fig. 1. The geometry of an IPS observation.

shown this bimodal nature more clearly than observations at earth or further out [Schwenn, 1990]. This is because the fast and slow wind in the ecliptic plane interact forming contact surfaces, shocks and enhanced turbulence. The interaction increases with distance from the sun. Recent observations from Ulysses also show this bimodal character clearly because stream interactions become less important at higher latitudes [Phillips et al., 1994].

We can take advantage of this bimodal character in the analysis of IPS observations. The basic geometry of an IPS observation is sketched in Fig. 1. Turbulent density fluctuations act as a flow tracer. They cause phase modulation of the radio wave passing near the sun, and diffraction converts this phase modulation to intensity modulation as the wave continues to propagate to the earth. We measure the intensity diffraction pattern at two places on the earth and estimate the time delay between the pattern at the two stations. Since the intensity pattern is a random process we use a cross correlation analysis to estimate the time delay. The cross correlation function should resemble the autocorrelation but it should peak at the desired time lag. An example is shown in Fig. 2.

Recent IPS observations made at the EISCAT observatory in northern Scandinavia began to show unusual cross correlations with two distinct peaks during the spring of 1994. An example is shown in Fig. 3. We assume that this is caused by the superposition of fast and slow wind. To confirm this we have examined the underlying corona carefully to see if one could expect the line of sight to cross fast and slow regions. Indeed the line of sight crosses a coronal hole, where one would expect a fast stream, and continues across the heliospheric current sheet, where one would expect slow wind. We then fit a model to the cross correlation and estimate the fast and slow speeds independently. This estimation is quite good when the two peaks are distinct. However even if the slow peak is not resolved one can often fit the fast peak quite accurately. 


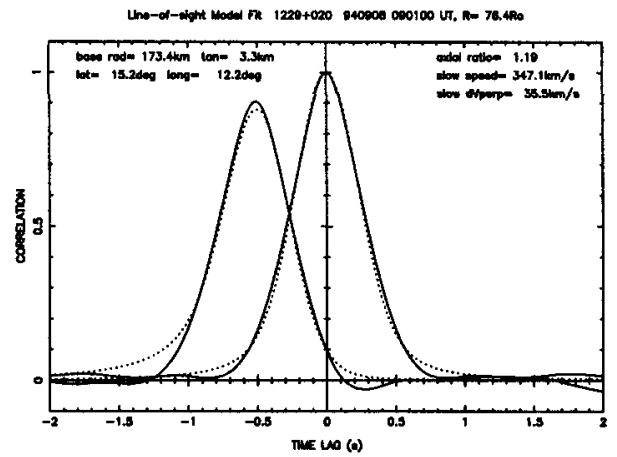

Fig. 2. Auto and cross correlation functions for an IPS observation at EISCAT on 940908. The estimated correlations are solid lines, the best fit model is overplotted with dashed lines. This observation is entirely in slow wind.

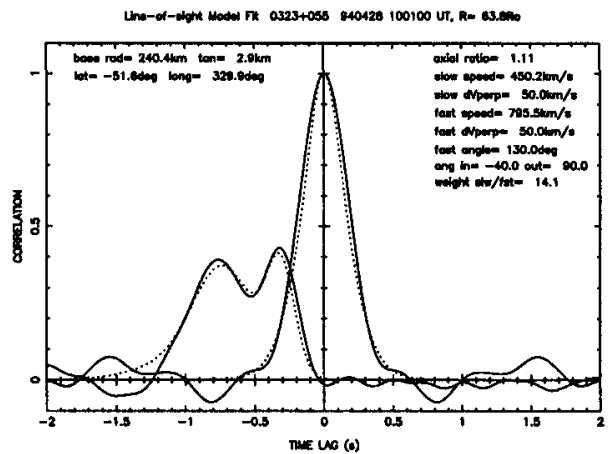

Fig. 3. Auto and cross correlation functions for an IPS observation at EISCAT on 940428. This observation has received equal contributions from fast and slow wind.

\section{Coronal Observations}

We used the soft X-ray maps from the Yohkoh spacecraft as a primary data source because they provide excellent resolution. We have assembled a synoptic map by using a strip from the center of the daily full disc map which is archived at the NSSDC. Such a map is shown in Fig. 4 for the rotation centered on the double peaked observation of Fig. 3. The gray scale is proportional to the $\log$ of the emission measure. The other primary data source are the polarized brightness observations made by $\mathrm{HAO}$ at Mauna Loa. Although these are limb observations, and thus have poorer longitude resolution, they depend only on the electron density and are thus easier to interpret. We have added contours representing the electron density at $1.7 \mathrm{R}_{\odot}$ derived from these white light observations. The position of the heliospheric current sheet, as estimated from the Wilcox Solar Observatory 


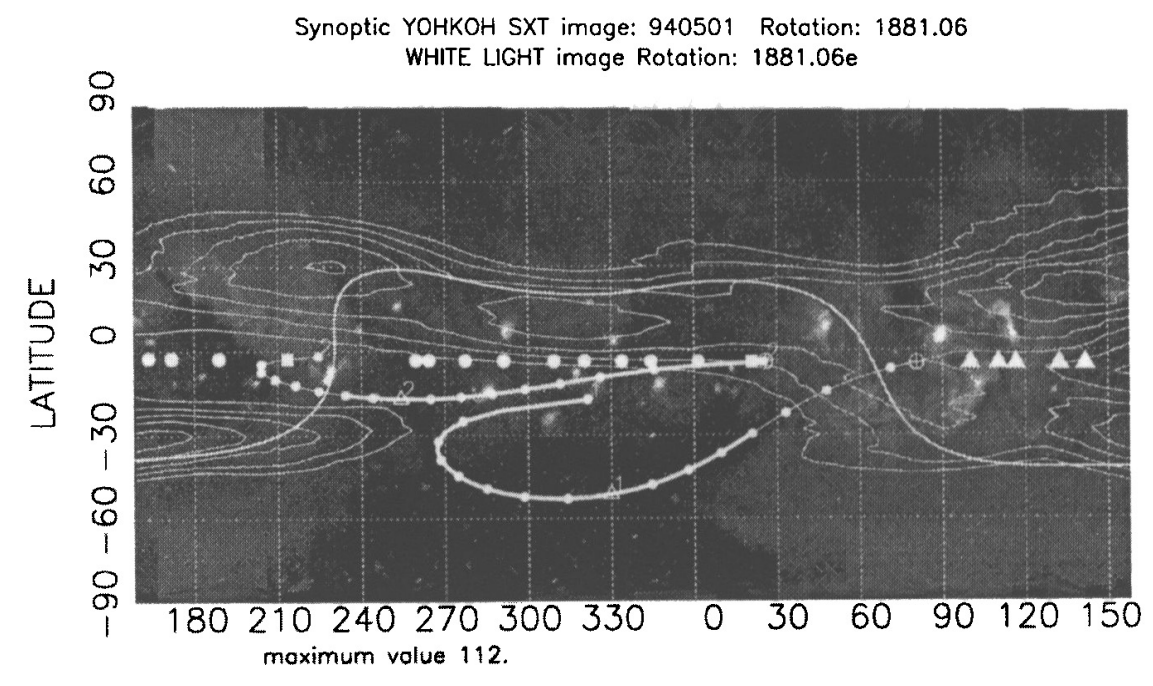

CARRINGTON LONGITUDE

Fig. 4. A synoptic map of soft X-ray emission from the Yohkoh spacecraft (gray scale) and the electron density at $1.7 \mathrm{R}_{\odot}$ derived from Mauna Loa white light observations (contours), overlaid with the magnetic neutral line from the Wilcox Solar Observatory (heavy line). The daily average IMP8 velocity measurements have been quantized into fast (circles), slow (triangles) and interaction regions (squares) and mapped back to the coronal base using a constant velocity trajectory. The IPS line of sight has also been mapped back and marked with small circles at $10^{\circ}$ intervals. The closest point of approach has been marked with a triangle. The earth is marked with an $\oplus$ symbol. The $10^{\circ}$ circles are connected with a light line in the slow wind and heavy line in the fast stream.

at Stanford University is overplotted as a heavy solid line. This is inferred from measurements of the photospheric magnetic field and a potential field mapping which is constrained to be radial at $2.5 \mathrm{R}_{\odot}$. Previous work indicates that the current sheet (where the radial component of the magnetic field reverses sign) should delineate the densest corona and the slowest solar wind. However it is known that the WSO estimates of the position of the current sheet tend to overestimate the latitude variation due to an underestimate of the polar field strength [Wilcox and Hundhausen, 1983].

We have mapped the IPS line of sight back to the coronal base at $10^{\circ}$ intervals using a simple radial-flow constant-velocity assumption and plotted the result on Fig. 4. The closest point to the sun is marked with an open triangle and a number. Each $10^{\circ}$ interval is marked with a small solid circle up to $80^{\circ}$ in the direction of the source and all the way back to the earth which is marked with an $\oplus$ symbol. The last $10^{\circ}$ is not plotted because there is very little contribution from such large solar distances. The IPS track numbered 1 corresponds to Fig. 3.

We have examined the corresponding IMP8 plasma data and identified 
three "modes"; fast wind, slow wind, and interaction regions. Daily averages have also been mapped back to the coronal base and overlaid it on Fig. 3 as large solid symbols. The fast wind is marked with a circle, the slow wind with a triangle and the interaction regions with a square.

\section{Model Fitting}

The IPS cross correlation function can be calculated, when the scattering is weak, from the distribution of velocity and density variance along the line of sight. The bimodal assumption allows us to simplify this since we need only specify the boundaries between the fast and slow wind and the conditions in these two "modes". In fact we do not need the exact density variance, but only the ratio of the variance in the slow wind to that in the fast wind. This ratio was found to exceed 10 by Bourgois and Coles [1992] for polar streams near the sun, and this was confirmed by Manoharan [1993]. The fact that the slow wind has a rather high density variance means that a relatively small part of the line of sight in slow wind can cause a large decrease in the apparent IPS velocity.

The IPS data in Fig. 3 is a good example. Here we used initial estimates of the fast and slow speeds of 800 and $400 \mathrm{~km} / \mathrm{s}$. Our initial estimate of the ratio of the slow to fast density variance was 15 , following the work of Coles, et al. [1995]. Examination of the IPS track marked 1 in Fig. 4 suggests that most of it lies in a large fast stream which is connected to the south polar hole. We assumed that only the region from the earth $\left(-73^{\circ}\right)$ to $-40^{\circ}$ was slow wind. The best fit correlations are overplotted as dashed lines on Fig. 3. In such cases, where both fast and slow peaks are resolved, one can estimate both speeds quite accurately. However it frequently happens that only one of the peaks is resolved and the other shows as a "shoulder" on the cross correlation.

Measurements of the solar wind velocity using multiple antenna cross correlation analysis have been made with a variety of instruments for nearly 30 years [Dennison and Hewish, 1967; Hewish and Symonds, 1969; Vitkevitch and Vlasov, 1970; Ekers and Little, 1971; Coles and Maagoe, 1972; Watanabe et al., 1973; Kojima et al., 1982; Scott et al., 1983; Bourgois et al., 1985; Armstrong et al., 1986]. Thus it may seem odd that double peaks in the cross correlations have not been discussed before. The reason for this is shown in Fig. 5. Here we have plotted the best fit to the example of Fig. 3 with the actual baseline used $(240 \mathrm{~km})$, and also with shorter baselines of $160 \mathrm{~km}$ and $80 \mathrm{~km}$. One can see that if the observation had been made with a shorter baseline the two peaks would not have been resolved. The important parameter is the ratio of the baseline to the spatial scale of the intensity diffraction pattern. Previous IPS observations have been made with much shorter effective baselines. The reason is that previous observations were 


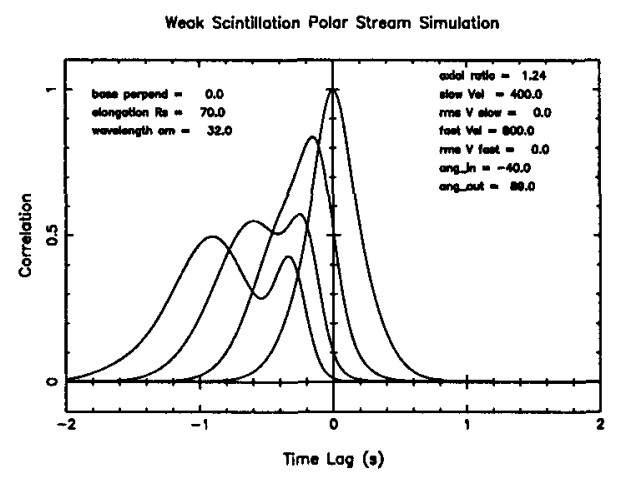

Fig. 5. Model correlation functions for the data of Fig. 3, and two examples of the expected IPS if baselines of $160 \mathrm{~km}$ and $80 \mathrm{~km}$ had been used.

made with three antenna systems in order to estimate the flow direction. This requires relatively short baselines to ensure that correlation is observed on all three baselines. However we now know that the flow is almost radial (at the distances where IPS observations can be made), so we can use a single very long radial baseline. There are a few existing observatories where such baselines can be employed. One of the best is EISCAT, but the Pietown and Los Alamos antennas of the VLBA have been used; the Jodrell Bank and Cambridge antennas of MERLIN could be used; and the Ooty and GMRT antennas could be used.

In order to see double-peaked cross correlations, and thus have an opportunity to correct for the line of sight integration one must satisfy four conditions. As mentioned above, the baseline must be substantially longer than the spatial scale of the diffraction pattern. In addition the scattering must be weak, since different contributions to the cross correlations are not additive in strong scattering and double peaks cannot occur. The line of sight must pass through both fast and slow wind, a common situation during the declining phase of solar activity, however it must not cross an interaction region. Since interaction regions build up with increasing solar distance, this latter condition implies that one must observe near the sun, yet in weak scattering. So one must use a relatively high frequency.

\section{The Velocity of the Fast Wind}

An attempt to measure the acceleration profile of the solar wind for comparison with theoretical models was made by Coles et al. [1990]. In that work the data were selected to measure the mean velocity at essentially the same latitude and longitude but different distances. The mean velocity was scaled up by $18 \%$ to remove the bias due to spherical divergence, but no attempt was made to correct for possible inclusion of both fast and slow wind in the 


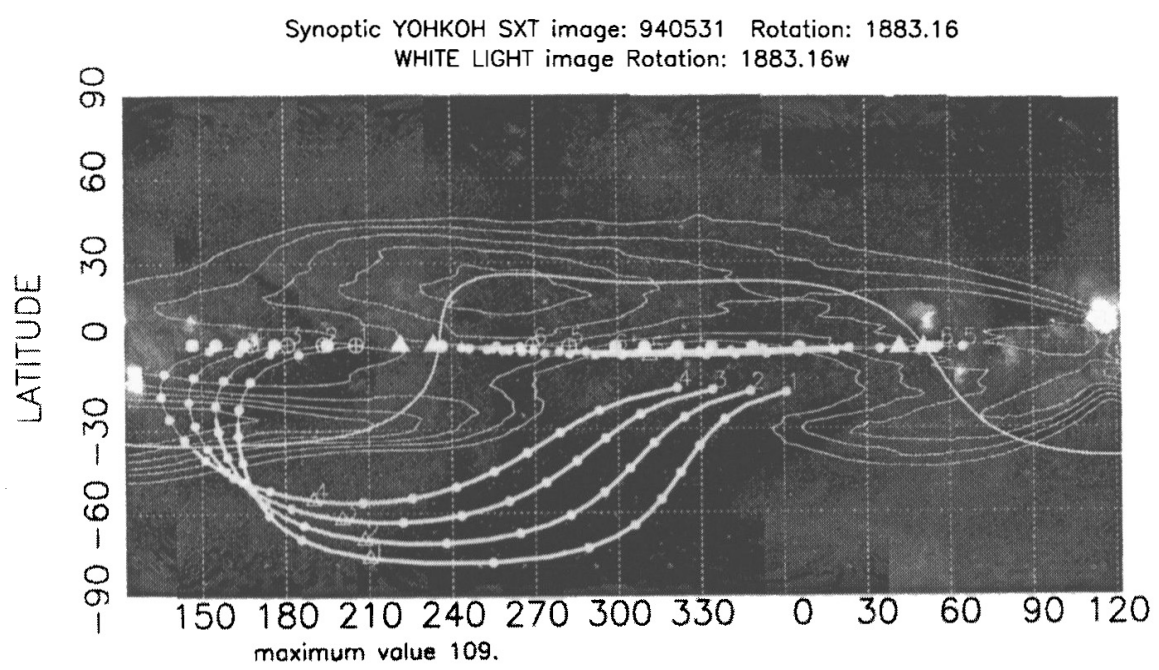

CARRINGTON LONGITUDE

Fig. 6. An overplot of coronal observations, IPS solar wind scattering region, and IMP8 solar wind measurements for rotation 1883 in the same format as Figure 3.

line of sight. This may be a reasonable approach when the line of sight is dominated by slow wind (which has a higher density variance), but is not very good when attempting to measure the speed of the fast polar stream. In this section we will present some preliminary measurements of the fast stream at a distance of 24 to $28 \mathrm{R}_{\odot}$.

Six observations of the south polar stream were made at EISCAT in late May of 1994, a time when the ULYSSES spacecraft was also in the south polar stream. The IPS track and coronal maps for this period are shown in Fig. 6. One can see that all six IPS tracks plotted are dominated by fast wind, and this is confirmed by inspection of the cross correlations. However all six observations have a significant contribution from the slow wind. A typical example is shown in Fig. 7. This comes from the track marked 1 on Fig. 6. The mean speed derived from the intersection of the auto and cross correlations is $585 \mathrm{~km} / \mathrm{s}$ which should be corrected to $690 \mathrm{~km} / \mathrm{s}$ for spherical divergence. In this case the contribution of the slow wind has reduced the mean speed $195 \mathrm{~km} / \mathrm{s}$ below that of the fast stream! The various velocity estimators for the six IPS observations shown are given in Table 1.

During this period the ULYSSES spacecraft was at similar latitudes in the south polar stream and measured a very stable speed between 750 and $800 \mathrm{~km} / \mathrm{s}$ at a distance of $630 \mathrm{R}_{\odot}$. Thus we see an apparent deceleration of the solar wind from 25 to $630 \mathrm{R}_{\odot}$. Of course it is possible that the solar wind actually does decelerate, but it is also possible that the IPS speed does 


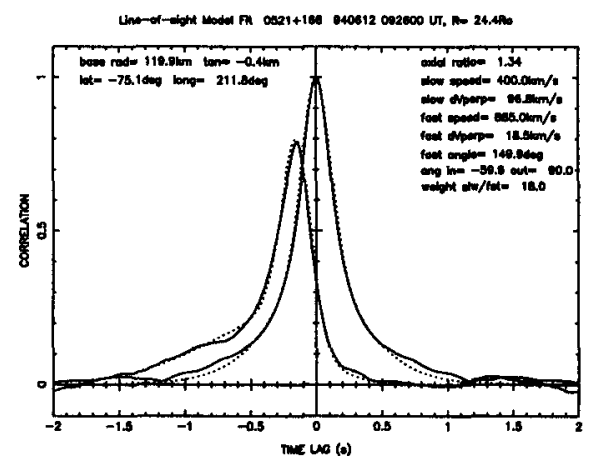

Fig. 7. Correlation functions and model fit for the IPS track marked 1 on Fig. 6

\section{TABLE I}

Velocity estimators for the IPS measurements of the south polar hole in rotation 1883. The peak velocity $V_{p k}$ has been used as a measure of the fastest speed. The midpoint (at $50 \%$ ) velocity $V_{m d}$ has been widely used as a measure of the mean velocity. The intersection velocity $V_{i n}$ is a good measure of the mean velocity when the velocity distribution is a single peaked monotonically decreasing function. The $V_{\text {fast }}$ and $V_{\text {slow }}$ velocities are the results of the best-fit bimodal model described in this work. The angle subtended by the fast stream at the Sun is also given. Negative angles are in the direction of the earth.

\begin{tabular}{lcccccccccc}
\hline & Date & Source & $\begin{array}{c}\mathrm{R}_{\text {min }} \\
\mathrm{R}_{\odot}\end{array}$ & $\begin{array}{c}\mathrm{V}_{\mathrm{pk}} \\
\mathrm{km} / \mathrm{s}\end{array}$ & $\begin{array}{c}\mathrm{V}_{\mathrm{mp}} \\
\mathrm{km} / \mathrm{s}\end{array}$ & $\begin{array}{c}\mathrm{V}_{\mathrm{in}} \\
\mathrm{km} / \mathrm{s}\end{array}$ & $\begin{array}{c}\mathrm{V}_{\text {fast }} \\
\mathrm{km} / \mathrm{s}\end{array}$ & $\begin{array}{c}\mathrm{V}_{\text {slow }} \\
\mathbf{k m} / \mathrm{s}\end{array}$ & $\begin{array}{c}\theta_{\text {fast }}\left({ }^{\circ}\right) \\
\text { from }\end{array}$ & to \\
\hline 1. & 940612 & $0521+166$ & 24.4 & 822 & 662 & 585 & 885 & 400 & -60 & 90 \\
2. & 940613 & $0521+166$ & 25.0 & 842 & 719 & 603 & 885 & 400 & -58 & 90 \\
3. & $\mathbf{9 4 0 6 1 4}$ & $0521+166$ & 25.9 & 842 & 681 & 591 & 885 & $\mathbf{4 5 0}$ & -41.5 & 90 \\
4. & 940615 & $0521+166$ & 27.4 & 828 & 475 & 544 & 885 & 450 & -37.5 & 90 \\
5. & 940606 & $0431+206$ & 24.7 & 790 & 651 & 623 & 850 & 450 & -20 & 60 \\
6. & 940607 & $0431+206$ & 28.2 & $\mathbf{7 5 0}$ & 629 & 581 & 850 & 450 & -15 & 70 \\
\hline
\end{tabular}

not represent the flow speed. The IPS speed is the velocity of the density fluctuations which are acting as the flow tracer. If these fluctuations are due to outwards propagating waves, then IPS will measure the sum of the flow velocity and the wave velocity. Since both the sound speed and the Alfven speed decrease rapidly with distance this apparent deceleration may well be caused by waves. This seems particularly likely in the fast wind, which we know to be dominated by outwards propagating waves. 


\section{Summary and Conclusions}

We have seen that IPS measurements of the solar wind speed may be severely affected by the line of sight integration, an effect that they share with most remote sensing observations. This problem is particularly acute when measuring the fast stream near the sun because the fast wind has a much smaller density variance than the slow wind. However if IPS observations are made with a sufficiently long baseline it is possible to resolve the fast and slow wind and estimate the two speeds independently.

The separation technique can only be used when the scattering is weak, because the cross correlation is not a simple line of sight integral in strong scattering. This severely limits the closest one can measure to the sun. EISCAT, for example, cannot measure inside of about $16 R_{\odot}$ over the poles at solar minimum or $30 \mathrm{R}_{\odot}$ in the equatorial regions because it cannot observe at frequencies above $933 \mathrm{MHz}$. The VLBA is particularly useful in this respect because it can operate at higher frequencies and will be able to measure as close as $4 \mathrm{R}_{\odot}$ at $8 \mathrm{GHz}$.

The separation technique cannot be used much further from the sun than $100 \mathrm{R}_{\odot}$ because interactions build up outside this distance and a bimodal model is no longer be useful. It may be possible to model the interaction regions, but it will be a more complex problem.

Preliminary results indicate that the apparent speed of the fast stream which is known to be between 750 and $800 \mathrm{~km} / \mathrm{s}$ at $630 \mathrm{R}_{\odot}$, has reached nearly $900 \mathrm{~km} / \mathrm{s}$ by a distance of $25 \mathrm{R}_{\odot}$. However the apparent speed may be the flow speed plus a wave speed so the IPS measurement does not necessarily indicate a deceleration.

\section{Acknowledgements}

I would like to acknowledge the assistance of my colleagues Russ Grall and Mike Klinglesmith at UCSD; Jussi Markkanen at EISCAT; and Andy Breen and Phil Williams at Aberystwyth, who helped make the observations, develop the analysis procedures and reduce the data. Todd Hoeksema provided the inferred magnetic field data from the Wilcox Solar Observatory. David Sime provided white light data from the Mauna Loa Solar Observatory. Alan Lazarus provided the reanalyzed IMP8 data. The 1994 EISCAT observations were made under the U.K. observing time allocation. The soft X-ray data is from the Yohkoh mission of ISAS, Japan. The X-ray telescope was prepared by the Lockheed Palo Alto Research Lab, the National Astronomical Observatory of Japan and the University of Tokyo, with support of NASA and ISAS. Our work was partially supported by the U.S. National Science Foundation (under grant ATM-9008608). 


\section{References}

Axford, W. I., this volume, 1995.

Axford, W. I. and J. F. McKenzie, in preparation, 1995.

Armstrong, J. W., W. A. Coles, M. Kojima and B. J. Rickett, Solar wind observations near the sun, in The Sun and the Heliosphere in Three-Dimensions, ed. R. G. Marsden (North Holland: Reidel) 1986.

Bourgois, G., W. A. Coles, G. Daigne, J. Silen, T. Turunen and P. J. Williams, Measurements of the solar wind velocity with EISCAT, Astron. Astrophys. 144, 452-462, 1985.

Bourgois, G. and Coles, W. A., Solar cycle changes in the turbulence level of the polar stream near the sun, In em Solar Wind Seven, ed. E. Marsch and R. Schwenn, Pergamon:Oxford,p 158, 1992.

Coles, W. A. and S. Maagoe, Solar wind velocity from IPS observations, J. Geophys. Res. (Lett.), 77, 5622, 1972.

Coles, W. A., R. Esser, U.-P. Løvhaug, and J. Markkanen, Comparison of solar wind velocity measurements with a theoretical acceleration model, J. Geophys. Res., 96, $13849,1991$.

Coles, W. A., R. R. Grall, M. T. Klinglesmith and G. Bourgois, J. Geophys. Res., submitted, 1995.

Dennison, P. A. and Hewish, A., The solar wind outside the plane of the ecliptic, Nature 213, 343-346, 1967.

Ekers, R. D. and L. T. Little, The motion of the solar wind close to the sun, Astron. Astrophys. 10, 310-316, 1971.

Hewish A. and M. D. Symonds, Radio investigation of the solar plasma, Planet. Space Sci. 17, 313, 1969.

Kojima, K., Y. Ishida, K. Maruyama and T. Kakinuma, An observation system of interplanetary scintillation at UHF, Proc. Res. Inst. Atmospherics of Nagoya Univ. 29, 61, 1982.

Manoharan, P. K., Three-dimensional structure of the solar wind: variation of density with the solar cycle, Solar Phys. 148, 153-167, 1993.

Marsch, E. and C.-Y. Tu, Spectral and spatial evolution of compressive turbulence in the inner solar wind, J. Geophys. Res. 95, 11945, 1990.

Phillips, J. L., A. Balogh, S. J. Bame, B. E. Goldstein, J. T. Gosling, J. T. Hoeksema, D. J. McComas, M. Neugebauer, N. R. Sheeley, Jr., and Y.-M. Wang, ULYSSES at $50^{\circ}$ south: Constant immersion in the high-speed solar wind, Geophys. Res. Lett. , in press, 1994.

Schwenn, R., The large scale structure of the interplanetary medium, In Physics of the Inner Heliosphere, 1: eds. R. Schwenn and E. Marsch (Springer-Verlag, Heidelberg), 99-181, 1990.

Scott, S. L., W. A. Coles, and G. Bourgois, Solar wind observations near the Sun using interplanetary scintillation, Astron. Astrophys. 123, 207, 1983.

Vitkevitch V. V. and V. I. Vlasov, Radio astronomical investigation of the drift of the inhomogeneous interplanetary plasma, Sov. Astron. A.J. 13, 669-676, 1970.

Watanabe, T., T. Kakinuma, M. Kojima and K. Shibasaki, Solar wind disturbance detected by the interplanetary scintillation of radio sources in early August 1972, $\mathrm{J}$. Geophys. Res. 78, 8364-8366, 1973.

Wilcox J. M. and A. J. Hundhausen, Comparison of heliospheric current sheet structure obtained from potential magnetic field computations and from observed polarized coronal brightness, J. Geophys. Res. 88, 8095, 1983. 